during a six week period despite maintaining a stable weight. The cause of these disturbances seemed to be located at or above the level of the hypothalamus in four patients and the pituitary in three, and one patient had polycystic ovaries. No patients suffering pituitary dysfunction had a history of anorexia nervosa.

The ovarian ultrasound scan confirmed the test findings in all but one patient (case 11), in whom the pituitary disturbance may have been of insufficient duration to cause a change in ovarian morphology.

Though interpretation of the luteinising hormone releasing hormone test is not absolutely reliable, this study shows that anovulation and menstrual disturbance are common in women with bulimia of normal body weight and that anovulation is not associated with a consistent hormonal pattern. Possible causes of these disturbances include gross fluctuations in carbohydrate intake, disturbances of fluid and electrolyte balance caused by self induced vomiting and laxative abuse, or emesis leading to excessive dopaminergic brain activity.

Dr Evans received a grant from the Alcohol Education and Research Council. We thank Dr J Nesbitt and the chemical pathology department, St George's Hospital, for the hormonal assays and the Special Trustees of St George's Hospital for financial support.

1 Crisp AH, Stonehill E. Relation between aspects of nutritional disturbance and menstrual activity in primary anorexia nervosa. $\mathrm{Br}$ Med f 1971;iii: 149-51.

menstrual activity in primary anorexia nervosa. Br Med 1971 , in. 149 -51. amenorrhoea. $\mathcal{f}$ Obstet Gynaecol Brit Commonwealth 1972;79:874-82.

3 Bergh T, Nillins SJ, Wide L. Serum prolactin and gonadotrophin levels before and after luteinising hormone releasing hormone in the investigation of amenorrhoea. Br J Obstet Gynaecol 1978;85:945-56.

4 Franks S, Adams J, Mason H, Polson D. Ovulatory disorder syndrome. Clin Obstet Gynaecol 1985;12:605-32.

(Accepted 8 June 1988)

\section{The closed eyes sign: an aid to diagnosing non-specific abdominal pain}

\section{Derek W R Gray, J Michael Dixon, Jack Collin}

Nuffield Department of Surgery, University of Oxford, John Radcliffe Hospital, Oxford OX39DU Derek W R Gray, FRCS, clinical lecturer J Michael Dixon, FRCS, registrar

Jack Collin, FRCS, clinical reader

Correspondence to: Mr Gray.
In $45 \%$ of patients attending hospital with acute abdominal pain no diagnosis is made ${ }^{1}$ and the term non-specific abdominal pain is used as a convenient diagnostic label. Probably patients with non-specific abdominal pain are in reality suffering from a wide range of conditions. Psychological factors may influence this pain or be the cause of it in some cases, and it would be helpful to be able to recognise physical signs pointing to a diagnosis of a psychosomatic condition. We noticed that patients with non-specific abdominal pain often keep their eyes closed during abdominal palpation whereas patients with organic disease usually keep their eyes open. Furthermore, the closed eyes are often associated with an expression inappropriate to a patient with pain. ${ }^{3}$ To test this observation we performed a prospective clinical study in patients admitted to hospital with abdominal pain.

\section{Patients, methods, and results}

We studied 158 consecutive patients complaining of abdominal pain admitted as an emergency to this department over six months. Patients with abdominal pain after trauma or recent surgery were excluded. The clinical details on admission were recorded on a standardised chart. During abdominal palpation the patient's eyes were carefully observed and the position of the eyelids recorded when abdominal tenderness were elicited. The findings were not used in subsequent management. After discharge from hospital all patients were seen at least once in the outpatient clinic, and the final diagnosis was recorded.

Of the 158 patients, 53 had a final diagnosis of appendicitis (median age 26 (range 11-89), male:female

Numbers of patients who closed their eyes during abdominal palpation

\begin{tabular}{lcccccccc}
\hline & \multicolumn{3}{c}{ No of patients observed } & & \multicolumn{3}{c}{ No (\%) who closed their eyes } \\
\cline { 2 - 3 } \multicolumn{1}{c}{ Diagnosis } & Total & Male & Female & & Total & Male & Female \\
\hline Appendicitis & 53 & 31 & 22 & & $2(4)$ & 2 & \\
Other disease & 38 & 20 & 18 & & $4(11)$ & & 4 \\
Non-specific abdominal pain & 67 & 25 & 42 & & $22(33)$ & 3 & 19
\end{tabular}

ratio of $1 \cdot 4: 1) ; 38$ had a variety of organic illnesses (median age 61 (17-88), male:female ratio $1 \cdot 1: 1$ ); and 67 had non-specific abdominal pain (median age 20 (11-75), male:female ratio $0 \cdot 6: 1$ ). Patients with appendicitis or non-specific abdominal pain were significantly younger than those with alternative diagnoses $(\mathrm{p}<0.06$, Mann-Whitney $U$ test), but there was no significant difference in age between patients with appendicitis and those with non-specific abdominal pain. The proportion of female patients was significantly higher among patients with non-specific abdominal pain than among those with appendicitis $\left(\chi^{2}=5.33, p<0.05\right)$. The table shows the proportions of patients who closed their eyes during abdominal palpation; the difference between the group with non-specific abdominal pain and the other groups was significant $\left(\chi^{2}=13 \cdot 1, \mathrm{p}<0.01\right)$. The predictive power of a positive result in the "closed eyes test" was 79\% and the predictive power of a negative result was $65 \%$.

\section{Comment}

Abdominal palpation to elicit tenderness is an unpleasant procedure endured by patients in the hope that it will help the doctor make a diagnosis. Voluntary guarding occurs when the patient sees the doctor's hand near the tender area. This phenomenon suggests that the natural reaction of a patient with genuine abdominal tenderness is to watch the doctor's hand carefully to avoid unnecessary pain. This eyes open response is to be expected in patients with abdominal tenderness secondary to organic disease. Our results showed that many patients with non-specific abdominal pain closed their eyes during abdominal palpation. One explanation for this is that they were aware (either consciously or unconsciously) that palpation would not produce severe pain, which implies that the pain had a psychological aetiology. There is some evidence that psychological causes contribute to non-specific abdominal pain. ${ }^{45} \mathrm{We}$ believe that this is the first description of a physical sign that may identify patients with non-specific abdominal pain due to psychological causes.

1 Wilson DH, Wilson RG, Walmsley RG, Horrocks JC, DeDombal FT Diagnosis of acute abdominal pain in the accident and emergency department. BrF S urg 1977;64:250-4

2 Gray DW, Collin J. Non-specific abdominal pain as a cause of acute admission to hospital. Br F Surg 1987;74:239-42.

3 Collin J, Gray DW. The eyes closed sign. Br Med f 1987;295:1656.

4 Ingram PW, Evans G. Right iliac fossa pain in young women. $\mathrm{Br}$ Med $\mathcal{J}$ 1965;ii: 149-51.

5 Joyce PR, Bushnell JA, Walshe JW, Moreton JB. Abnormal illness behaviou and anxiety in non-organic abdominal pain. Br f Psychiatry 1986;149:57-62. 